\title{
Los MOOCs en la enseñanza superior: una tarea pendiente
}

\author{
Hipólito VIVAR ZuRITA \\ hvivar@ccinf.ucm.es \\ Universidad Complutense de Madrid \\ Raquel VinAder SEgura \\ rvinader@ucm.es \\ Centro de Estudios Superiores Felipe II (UCM) \\ Natalia ABuín VenCES \\ nabuinve@ucm.es \\ Universidad Complutense de Madrid
}

Recibido: 23 de julio de 2014

Aceptado: 27 de octubre de 2014

\section{Resumen}

La llegada de las TIC a la educación ha puesto de moda un nuevo tipo de cursos online basados en la gratuidad y la apertura de contenidos a todo tipo de usuarios: los Massive Online Open Courses (MOOCs). La gran aceptación de esta nueva modalidad de enseñanza hace que las principales universidades de todo el mundo estén creando plataformas para ofrecer este tipo de cursos, sin la existencia de un modelo de negocio que los haga rentables y con una financiación a fondo perdido por parte de sus impulsores. El objetivo del presente trabajo es analizar las expectativas y el nivel de aceptación de este tipo de cursos mediante la realización de un experimento de campo, basado en la realización de un MOOC por parte de una muestra de alumnos de comunicación.

Palabras clave: MOOCs, TIC en educación, enseñanza superior, innovación educativa, docencia virtual.

\section{Higher Education MOOCs: a Work in Progress}

\begin{abstract}
The advent of ICT to education has brought back into fashion a new type of online courses based on free and open access to all kind of users: Massive Online Open Courses (MOOCs). The popularity of this new teaching mode does that major universities and academic institutions around the world are creating platforms to offer this kind of courses, without the existence of a business model that can make them profitable by its promoters. The aim of this paper is to analyze the expectations and level of acceptance of such courses by conducting a field experiment, based on the study of a MOOC by a sample of students of communication.
\end{abstract}

Keywords: MOOCs, ICT in Education, Higher Education, Educational Innovations, Virtual Teaching.

\section{Referencia normalizada}

VIVAR ZURITA, Hipólito; VINADER SEGURA, Raquel; y ABUÍN VENCES, Natalia (2015): "Los MOOCs en la enseñanza superior: una tarea pendiente". Estudios sobre el Mensaje Periodístico. Vol. 21, Núm. 1 (enero-junio), págs.: 629-642. Madrid, Servicio de Publicaciones de la Universidad Complutense.

Sumario: 1. Introducción y estado de la cuestión; 1.1. Los MOOCs como herramienta de innovación educativa; 1.2. Principales iniciativas nacionales e internacionales; 1.2.1. Udacity; 1.2.2. edX; 1.2.3. Coursera; 1.2.4. OpenupED; 1.2.5. Miriadax; 1.3. Modelo de negocio. 2. Metodología; 2.1. Diseño de la investigación; 2.1.1. Elección de la muestra; 2.1.2. Selección de la muestra; 2.1.3. Pre-test; 2.1.4. Diseño experimental; 2.1.5. Post-test. 3. Discusión de los resultados. 4. Conclusiones. 5. Referencias bibliográficas. 


\section{Introducción y estado de la cuestión}

Los MOOCs comenzaron a ganar notoriedad en 2001, momento en el que numerosas instituciones educativas se unieron para crear plataformas para ofertar este tipo de cursos. Udacity, Coursera, Edx o Miriadax son algunos de los proyectos más importantes impulsados por universidades como Harvard o el Instituto Tecnológico de Masachusets (MIT).

Los defensores de los MOOCs afirman que este tipo de cursos llegan para revolucionar uno de los pocos sectores que no ha incrementado su productividad desde sus orígenes: el educativo.

Si nos remontásemos al siglo XVIII y analizásemos cada uno de los sectores de la sociedad desde la Revolución Industrial, nos daríamos cuenta de que la educación es uno de los pocos que no ha incrementado su productividad, a pesar de que la tecnología lo posibilita. Los avances han permitido que las fábricas produzcan cada vez más y con el empleo de menos tiempo y esfuerzo gracias a las máquinas. Sin embargo, el sector educativo sigue empleando las mismas técnicas de enseñanza que se empleaban en la Grecia Clásica: clases magistrales, exámenes y evaluaciones.

Actualmente, en pleno proceso de implantación del Espacio Europeo de Educación Superior y con nuestro país sumido en una profunda crisis económica, las aulas tienen cada vez más alumnos y menos profesores. Cabe preguntarse entonces si las TIC podrían ayudarnos a atravesar esta etapa de profundos cambios y mejorar los resultados del sistema educativo: satisfacer las necesidades de conocimiento de más alumnos consumiendo menos esfuerzo y con escasos recursos económicos.

La respuesta puede estar en los denominados MOOCs, cursos online, gratuitos, sin límite de alumnos y dirigidos por los profesores más prestigiosos del mundo.

\subsection{Los MOOCs como herramienta de innovación educativa}

Los Massive Online Open Courses (MOOCs) alcanzaron su popularidad en 2011, aunque la idea surgió diez años antes. Tienen su origen en el concepto de Social Learning que hace referencia a la posibilidad de aprender través de la interacción con otros individuos. Según Atkins et alt (2007) las tecnologías Web desarrolladas en las dos últimas décadas han provocado que la oferta educativa a través de la red se haya multiplicado. La Web 2.0 permite a los usuarios interactuar con la información y el conocimiento de una manera más activa en una gran variedad de formatos y actividades educativas (Seely, 2008).

En este entorno 2.0 en el que los internautas quieren ser los protagonistas y compartir información y conocimientos surgen los MOOCs, cuyo germen fueron las plataformas Fhatom y AllLearn.

Pero, es en 2011 cuando este tipo de formatos alcanzan su popularidad gracias al curso Introduction to Artificial Intelligence ofrecido por Sebastian Thrun y Peter Norvig en la Universidad de Stanford a través de una compañía startup llamada Know Labs (actualmente Udacity), en el que se matricularon más de 160.000 personas. Tras esta iniciativa empezaron a aparecer diferentes plataformas como Coursera o edX.

Según lo expuesto, este tipo de cursos son una evolución de la educación en abierto a través de internet y deben tener cuatro características fundamentales: 
- Masivos: deben ir dirigidos a un número ilimitado de alumnos.

- Abiertos: puede inscribirse cualquier persona, independientemente de su formación previa. Además, el contenido es abierto; está disponible online. Por último, el concepto "abierto" hace referencia a que son gratuitos, es decir, que cualquier persona puede recibir formación impartida por los profesores de las universidades más exclusivas del mundo.

- Online. Se imparten única y exclusivamente a través de la Red por lo que son accesibles globalmente.

- Son cursos. Esto quiere decir que hay un profesor-tutor, unas fechas de inicio y fin y un sistema de evaluación.

Tal y como se ha apuntado anteriormente, los MOOCs están pensados para tener una audiencia masiva: cualquier individuo desde cualquier parte del mundo puede inscribirse. Tal y como se ha apuntado anteriormente algunos han sobrepasado los 150.000 alumnos.

Una cifra así sólo puede ser gestionada de forma virtual. Sin embargo es muy complicado, por no decir imposible, proporcionar una atención personalizada como la que se ofrece en la enseñanza presencial. Aunque las TIC nos permiten distribuir información de manera masiva a través del mail o de los foros, la interacción personal y la conversación con el alumno se hace inviable e imposible en estos entornos.

Además de la comunicación, la masificación plantea otro importante problema: la evaluación. Aunque se están desarrollando diferentes programas informáticos diseñados para evaluar, corregir y proporcionar feedback al alumno, este problema limita mucho el tipo de ejercicios que pueden plantearse.

La segunda característica de estos cursos es que son "online". Las ventajas de este sistema radican en que son accesibles para personas que por trabajo o cargas familiares no disponen de tiempo para desplazarse a un centro educativo. Además, son globales, esto es que cualquier persona de cualquier parte del mundo puede acceder a esos conocimientos. Cada alumno decide cuando y donde completar el curso, sin horarios y desde la comodidad de su domicilio.

Sin embargo la formación online también tiene sus desventajas. La tasa de abandono de los MOOCs es del 90\%. Esto se debe a que ante la inexistencia de horarios y la "ausencia" del profesor, a los alumnos les cuesta seguir una rutina formativa, más aún si estos cursos son gratuitos.

En tercer lugar hablamos de cursos abiertos, un concepto que implica múltiples acepciones: puede inscribirse cualquier persona, sin necesidad de credenciales y sin tener que demostrar un nivel mínimo de conocimientos para recibir la formación. Esto tiene una parte positiva y es que permite el acceso a cursos de élite a cualquier persona, sin embargo la parte negativa es que al no medir el nivel de los participantes ni requerir un perfil específico, la audiencia es heterogénea y se incrementa la tasa de abandono entre los que no poseen la base de conocimientos suficiente para seguir el curso.

Además el concepto "abierto" implica que el material del curso está completamente disponible a través de la red. Esto facilita el acceso de los usuarios a los contenidos pero plantea problemas con los derechos de autor de los materiales. 
Por otro lado, "abierto" significa gratis. La idea de que los MOOCs sean gratuitos ha supuesto un gran hito en el ámbito educativo ya que supone democratizar la educación de élite. Este es un gran paso, más aún en los países subdesarrollados donde hay muy pocas personas capaces de costearse un curso universitario, algo que puede contribuir enormemente al desarrollo económico de estos países. Sin embargo la gratuidad de los MOOCs es también su mayor problema, ya que no existe por ahora ningún modelo de negocio que los haga rentables y permita financiarlos.

Por último, son "cursos", el alumno recibe una formación, adquiere unas competencias y es evaluado por ello. El gran problema en este sentido es el de la evaluación y la certificación. Muchos alumnos se matriculan es estos cursos por el mero placer de aprender, sin embargo otros lo hacen porque necesitan adquirir determinadas competencias para mejorar en su trabajo o conseguir uno nuevo por lo que necesitan certificar sus conocimientos y la mayoría de los MOOCs no ofrecen esta posibilidad.

\subsection{Principales iniciativas nacionales e internacionales}

Los MOOCs están revolucionando el concepto de la educación superior y la formación online. Pero, como comentábamos no todos los MOOC son iguales. De hecho, no existe unanimidad a la hora de afrontar su estudio.

La clasificación más extendida es la que divide estos cursos atendiendo a la teoría o corriente de aprendizaje correspondiente. Así, puede hablarse de cMOOC, para referirse a los conectivistas, y de xMOOC para los no conectivistas. Los cMOOCs son cursos gratuitos y verdaderamente accesibles que buscan el aprendizaje basándose fundamentalmente en la teoría conectivista. Éste se consigue a través de la interacción con otros estudiantes (a través de blogs, feeds RSS) y son precisamente estas relaciones el mayor valor añadido de estos cursos. De hecho, en estos casos la certificación pasa a un segundo plano. Se trata de facilitar la creatividad y el trabajo colaborativo en red.

Por su parte, los xMOOCs, no conectivistas, se centran en el contenido que se ofrece únicamente a través de la página web y que permite gestionar grandes cantidades de alumnos inscritos. En este caso, la colaboración es más rígida y la superación del curso se mide a través de la evaluación por test o trabajos. Udacity, EdX y Coursera son algunos ejemplos de xMOOCs.

\subsubsection{Udacity}

Cuando los profesores de la Universidad de Stanford, Thrun y Sokolsky, crean Udacity en 2011 buscan reinventar la educación del siglo XXI haciendo que ésta fuera "accesible, asequible, agradable, y muy eficaz para el mundo". Así, los MOOCs permiten satisfacer un "derecho humano básico" con el que los estudiantes pueden adquirir conocimientos sobre diversas materias pero también avanzar en el desarrollo profesional de los trabajadores en activo (Carr, 2012). Se trata, en palabras de los creadores, de "construir un puente entre las habilidades del mundo real, una educación pertinente y el empleo".

Actualmente Udacity tiene registrados 160.000 alumnos de 190 países distintos. Esta plataforma sin ánimo de lucro ofrece actualmente casi una treintena de cursos clasificados en cinco categorías (Negocios, Ciencias de la Computación, Diseño, Matemáti- 
cas y Ciencia) y tres niveles de formación (Principiante, Intermedio y Avanzado). Al mismo tiempo, tiene asociadas 611 comunidades de usuarios, los udacians, repartidas por 576 ciudades del mundo, con los que busca revitalizar las comunidades locales.

\subsection{2. edX}

En mayo de 2012 la Universidad de Harvard y el Instituto Tecnológico de Massachusetts (MIT) anuncian la inversión de sesenta millones de dólares para la creación de edX (Kolovich, 2013), un proyecto cuyo principio rector era mejorar la educación en el aula, no suplantarla. Hoy, esta plataforma está respaldada por un consorcio formado por veinte universidades de todo el mundo.

A pesar de considerarse como una plataforma sin ánimo de lucro y de que los cursos que ofrecen sean gratuitos, algunos de ellos cobran una tarifa por certificación. El dinero recaudado se reinvierte, según esta iniciativa, en la mejora de la plataforma. Los certificados pueden ser de tres tipos:

- Honor Code: acredita gratuitamente la superación del curso pero no refleja la identidad del alumno.

- ID Verified Certificate: permite certificar que el alumno ha completado el curso, identificando al estudiante con su nombre y su foto. Este tipo de diploma es de pago y sólo está disponible en una selección limitada de cursos.

- Por último, el XSeries Certificate para aquellos estudiantes que haya superado una serie de cursos vinculados a una materia determinada.

\subsubsection{Coursera}

Esta plataforma cuenta actualmente con 447 cursos distribuidos en 23 categorías diferentes y, aunque se imparten dominantemente en inglés, también pueden cursarse en francés, español o chino.

La filosofía de este proyecto es permitir el acceso gratuito y mundial a la enseñanza para "capacitar a las personas con educación que mejoren sus vidas, las vidas de sus familias y las comunidades en las que viven". Para ello, cuenta con el apoyo de una gran cantidad universidades de todo el mundo.

Las cifras conseguidas por esta iniciativa son increíbles. Según sus propias fuentes a finales de 2013 contaba con 17 millones de alumnos inscritos procedentes de 190 países en sus 440 cursos.

Coursera también ofrece a las empresas una manera de colaborar en la formación continua de sus trabajadores. De este modo, las compañías pueden poner a disposición de sus recursos humanos una amplia variedad de cursos que, a cambio de una esponsorización, la plataforma se encargará de validar y certificar convenientemente. Se trata, por tanto, de un modelo de negocio alternativo que ayuda al mantenimiento de la plataforma.

\subsubsection{OpenupED}

La Unión Europea, concienciada en la importancia del conocimiento como derecho pero también como modo para salir de la crisis, no ha querido quedar atrás. Coordinada por la EADN (acrónimo en inglés para Asociación Europea de Universidades a 
Distancia) y respaldada por la Comisión Europea, crea OpenupED, cuyo objetivo es acercar una "educación que refleje los valores europeos, tales como la equidad, la calidad y la diversidad". Así, busca ofrecer un modelo de universidad abierta con materiales de auto-aprendizaje de alta calidad.

Cuenta con once universidades repartidas por diez países distintos: Francia, Italia, Lituania, Países Bajos, Portugal, Eslovaquia, España, Gran Bretaña, Rusia, Turquía e Israel. Inicialmente se lanza con 40 cursos disponibles en doce lenguas distintas y en estos momentos tiene 71 .

\subsubsection{Miriadax}

Por último, tenemos que hablar de Miriadax. Bajo el paraguas de Universia -una red de 1.242 universidades iberoamericanas-, y el apoyo tecnológico de Telefónica Learning Services -compañía especializada en ofrecer soluciones integrales de aprendizaje online-, constituye la apuesta española más importante dirigida a todo el mercado hispanohablante. Tiene un doble propósito: apoyar a los estudiantes universitarios para el perfeccionamiento de conocimientos durante su carrera y colaborar en la mejora del perfil profesional de aquellos que ya están trabajando. Para ello, ofrece cursos diseñados y organizados por veinte universidades en distintas áreas de conocimiento.

La estructura y funcionamiento es similar a las expuestas anteriormente pero profundiza en la dimensión más social de este tipo de educación. Los cursos cuentan con video-lecciones que se completan con actividades obligatorias y optativas. Se ofrecen dos tipos de certificaciones a los estudiantes:

- Certificado de participación, cuando se hayan completado el 75\% de las actividades obligatorias.

- Certificado de superación al entregar el 100\% de las tareas encomendadas.

Además, la plataforma entrega badges, una insignia virtual "que refleja una habilidad o logro conseguido". En este caso, por cada curso finalizado.

\subsection{Modelo de negocio}

Uno de los principales hándicaps con los que se encuentran este tipo de cursos es la inexistencia de un modelo de negocio.

Tal y como se ha apuntado anteriormente, esta fórmula de Social Learning tiene como condición esencial la gratuidad de la oferta formativa. El desafío se encuentra actualmente en combinar una oferta atractiva de cursos, que sirva a su vez para retroalimentar la imagen de la institución, con un sistema de financiación que permita cumplir con los objetivos anteriores.

Algunas iniciativas han apostado por implantar el cobro de una cantidad simbólica en concepto de matrícula. Un mecanismo que puede mejorar notablemente la rentabilidad de estos cursos. Con sólo cobrar un euro a cada alumno, un MOOC podría recaudar miles si tenemos en cuenta las increíbles cifras de alumnos matriculados que se han expuesto.

Otras plataformas de MOOCs son partidarias de ofrecer formación gratuita pero cobrar por un certificado que acredite los conocimientos. De este modo, el alumno 
podría incorporar este título a su currículo y con su aportación económica se sostendría el sistema para seguir ofreciendo formación gratuita. Este mecanismo permitiría la pervivencia de del espíritu original de los MOOCs.

No obstante, debemos resaltar una última vía para el desarrollo económico de estos cursos masivos online y es la que vincula a las instituciones educativas con el mercado profesional. Consistiría en ofrecer a las empresas este tipo de cursos, tal y como hace Coursera, como base de su catálogo de formación continua. De esta manera, universidad, empresa y trabajadores ganarían mediante esta alianza. La universidad obtendría reconocimiento y sustento económico del mercado profesional; las empresas mejorarían su plan de formación interna a un coste asequible y los trabajadores tendrían una formación especializada y certificada por instituciones de reconocido prestigio.

\section{Metodología}

La metodología empleada para la conducción del presente trabajo de investigación ha sido un experimento de campo, cuyo objetivo fundamental era analizar la percepción de los estudiantes sobre los MOOCs en un entorno real de aplicación y sin la mediación del profesor. De esta forma, y a través de dos encuestas estructuradas y de respuesta abierta y semi-abierta, se analizó la percepción y el conocimiento de alumnos universitarios sobre este tipo de cursos y posteriormente el grado de satisfacción de los mismos tras realizar uno de ellos.

\subsection{Diseño de la investigación}

La presente investigación se realizó en cinco fases: elección del curso, selección de la muestra, pre-test, diseño experimental y post-test.

\subsubsection{Elección de la muestra}

Tal y como se ha apuntado anteriormente el presente proyecto se sustenta en el desarrollo de un experimento de campo cuya base es la realización de un MOOC por parte de los sujetos objeto de estudio. El primer paso antes de acometer el experimento fue la elección del curso. El MOOC debía cumplir una serie de requisitos:

1. Estar abierto todo el año, es decir, no exigir su inicio o finalización en un plazo determinado, puesto que la conducción del estudio estaría circunscrita a esas fechas limitando mucho el trabajo de los investigadores y la ejecución de las encuestas que acompañan el presente proyecto.

2. La totalidad de los módulos debían poder ser superados en un plazo de cuatro semanas, para evitar que los sujetos objeto de estudio perdieran interés y se incrementara la tasa de abandono, ya de por si elevada en este tipo de cursos.

3. El tema del MOOC debía ser atractivo y orientado a la comunicación, ya que el experimento se iba a realizar con estudiantes de Comunicación Audiovisual y Publicidad de la Universidad Complutense de Madrid.

4. El idioma del curso debía ser el español, para conseguir una muestra de alumnos lo más amplia posible con el objetivo de que los resultados obtenidos tuvieran validez externa. 
De entre toda la oferta de MOOCs existente en nuestro país se eligió "El Nuevo Profesional Digital" puesto que era el único que cumplía todos los requisitos exigidos por el estudio. Este curso ha sido producido por AMETIC (Asociación Multisectorial de Empresas de la Electrónica, las Tecnologías de la Información y la Comunicación, de las Telecomunicaciones y de los Contenidos Digitales) y respaldado por la Secretaría de Estado de Empleo y el Fondo Social Europeo para la promoción y el fomento de las competencias exigidas por la economía digital, por lo que la calidad y rigor del curso están garantizados.

\subsubsection{Selección de la muestra}

El experimento se llevó a cabo con alumnos de Comunicación Audiovisual y Publicidad de la Universidad Complutense de Madrid, procedentes de los campus Ciudad Universitaria y Felipe II, por lo que se trata de un muestreo casual o incidental. Se eligió este tipo de muestreo porque el experimento diseñado requiere un seguimiento exhaustivo de los sujetos objeto de estudio, por lo que se decidió circunscribirlo a los alumnos de los profesores participantes por la accesibilidad y la facilidad para comunicarse con ellos de los investigadores encargados de conducir el estudio.

Los profesores solicitaron voluntarios entre sus alumnos para realizar el curso y se apuntaron un total de 55 individuos: 43 mujeres y 12 hombres, de los cuales finalizaron el MOOC 44: 32 mujeres y 12 hombres.

\subsubsection{Pre-test}

Antes de iniciar la investigación y de enviar a los alumnos el enlace al curso se les pidió que respondieran un pre-test: un cuestionario estructurado de respuestas abiertas y semi-abiertas cuyo objetivo era medir el grado de conocimiento de los sujetos objeto de estudio sobre los Massive Online Open Courses y sus expectativas antes de realizar uno de ellos. Este cuestionario se diseñó con la herramienta Google Drive y se accedía al mismo a través de un link.

Este primer test estaba compuesto por dos partes claramente diferenciadas: la primera destinada a la identificación de los sujetos objeto de estudio. Los alumnos debían consignar una serie de datos personales que permitieran a los investigadores realizar un seguimiento individualizado, ya que sólo los que respondieran el pre-test recibirían el enlace al curso y podrían seguir colaborando en la investigación.

La segunda parte tenía por objetivo evaluar el conocimiento previo de los alumnos sobre los MOOCs y sus expectativas, y estaba compuesta por nueve preguntas, algunas de las cuales requerían de una respuesta abierta por parte delos sujetos objeto de estudio. El tiempo estimado de respuesta era de entre 5 y 10 minutos. Se diseñó de esta forma para evitar que los alumnos abandonaran el experimento y obtener una muestra lo más representativa posible.

Una vez elaborado el cuestionario se les envió el link a todos los alumnos que habían manifestado su interés en participar. Se les dio un plazo de 72 horas para responderlo, sólo podrían seguir adelante con la investigación y realizar el curso aquellos que cumplieran con este requisito, para evitar que las respuestas de los alumnos se dilataran en el tiempo lastrando el transcurso de la investigación y del propio experimento. 
La tasa de respuesta fue del 100\%, lo que pone de manifiesto el interés de los estudiantes por acceder al curso del que se les había hablado en las aulas.

\subsubsection{Diseño experimental}

Una vez obtenidas las respuestas al pre-test, se les envió el link al curso con una serie de instrucciones que debían seguir para poder completarlo con éxito:

- Debían registrarse en la plataforma.

- El plazo para realizarlo era de un mes desde la fecha de recepción del link.

- Tras la realización del curso debían enviar un justificante a los profesores mostrando la finalización de los seis módulos que componen el MOOC.

\subsubsection{Post-test}

Tras agotarse el plazo para realizar el curso, se les hizo llegar, sólo a los que habían enviado el justificante de haberlo terminado, el post-test: un cuestionario cuyo objetivo era conocer la impresión de los alumnos tras realizar un MOOC y su opinión sobre la utilidad de este tipo de cursos a la hora de potenciar la inserción laboral.

El cuestionario se dividió en tres partes claramente diferenciadas: datos personales, evaluación de la experiencia y desarrollo del MOOC.

La primera parte, idéntica en el pre-test, tenía por objetivo identificar a los alumnos para realizar un seguimiento de los que iban a completar todas las fases de la investigación.

La segunda estaba compuesta por tres ítems: una escala tipo Likert en la que se les pedía que puntuasen del 1 al 8 la experiencia general del curso y dos preguntas para medir las expectativas y el grado de adecuación de los contenidos del curso a los estudios que estaban cursando.

La tercera parte del cuestionario tenía por objetivo conocer los lugares, dispositivos y horarios preferidos para la realización del curso y estaba compuesto por 6 ítems, el último para saber si recomendarían este tipo de cursos a sus compañeros.

Igual que en el caso del pre-test se les dio un plazo de 72 horas para responderlo y cerrar la investigación. La tasa de respuesta fue del 100\% (de entre los que habían completado el curso).

Una vez completadas las tres fases se procedió al análisis estadístico de los datos y a la extracción de los resultados más relevantes derivados de la ejecución del presente diseño experimental.

\section{Discusión de los resultados}

El pre-test tenía, como se ha mencionado anteriormente, la función de conocer el perfil de los participantes y sus conocimientos previos sobre este modelo de enseñanza. El cuestionario inicial fue respondido por un total de 55 alumnos, de los cuales el 78\% eran mujeres frente al $22 \%$ de hombres. Mayoritariamente se trataban de estudiantes de Comunicación Audiovisual (64\%) procedentes de los campus ya mencionados: Ciudad Universitaria (58\%) y Felipe II (42\%). Para completar el perfil destacar que la mayor parte de los participantes no compagina sus estudios con un trabajo remunerado; únicamente el 16\% de los encuestados declaran compaginar las clases con una actividad profesional. 
Si analizamos los resultados obtenidos de los conocimientos previos sobre los MOOC, podemos observar que el $60 \%$ de los encuestados declara conocer este tipo de cursos, mientras que el $80 \%$ considera a priori que pueden ser de utilidad para complementar la docencia tradicional recibida en las aulas. No obstante, prácticamente la totalidad de los participantes (95\%) no había realizado ninguno antes de este experimento.

Únicamente tres estudiantes manifiestan haber realizado un curso de estas características, aunque al ser preguntados sobre los mismos, se observa el desconocimiento en la materia al no saber distinguir algunos cursos online de los MOOC.

Por último, se observa un importante desconocimiento de la oferta de MOOC tanto de la UCM como de otras universidades. Más del $90 \%$ declara no conocer los cursos ofertados por nuestros centros de enseñanza superior.

Realizado el curso seleccionado, se les solicitó responder a un cuestionario con el fin de evaluar la experiencia. De los 55 participantes iniciales que respondieron el pre-test, sólo 40 lograron terminar el curso y responder al post-test, por lo que la tasa de abandono fue del $20 \%$. El perfil medio del alumnado se mantiene con un predominio más significativo de los estudiantes de Comunicación (75\%) y se reparte equitativamente entre los dos campus. Se observa así que el abandono ha sido más significativo entre los estudiantes de Publicidad y Relaciones Públicas.

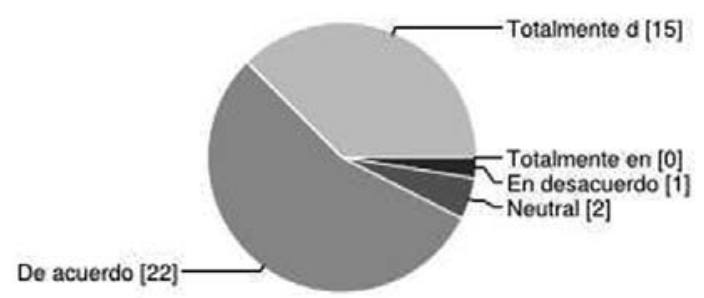

Gráfico 1: Grado de cumplimiento de las expectativas generadas por el curso.

Fuente: elaboración propia

La experiencia ha sido para los participantes altamente satisfactoria. E1 84\% de los participantes evalúa el curso con las puntuaciones más altas ( 7 y 8). Sólo un alumno lo califica con una nota por debajo del aprobado. Así, el 93\% de los encuestados declara que el MOOC seleccionado cumple con las expectativas iniciales.

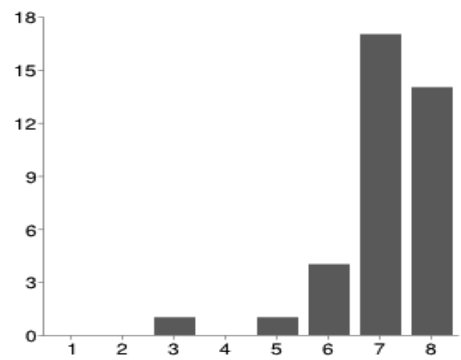

Gráfico 2: Valoración de la experiencia general del curso. Fuente: elaboración propia

Teniendo en cuenta la duración del curso, podemos ver que, si bien se encuentra una gran dispersión en la fecha de inicio, la mitad de los estudiantes finalizaron al 
borde del plazo límite asignado. E1 80\% de los participantes lo realizaron desde su casa, mientras que un número reducido lo hicieron desde la propia universidad $(10 \%)$, la biblioteca $(8 \%)$ o un cibercafé $(2 \%)$.

Al preguntar a los alumnos en qué horarios en los que realizaron el curso, propuesto, nos encontramos con una amplia variedad de respuestas. Como podemos ver en el siguiente gráfico, los participantes utilizaron todas las franjas horarias aunque claramente la tarde fue el horario más habitual para completar la formación.

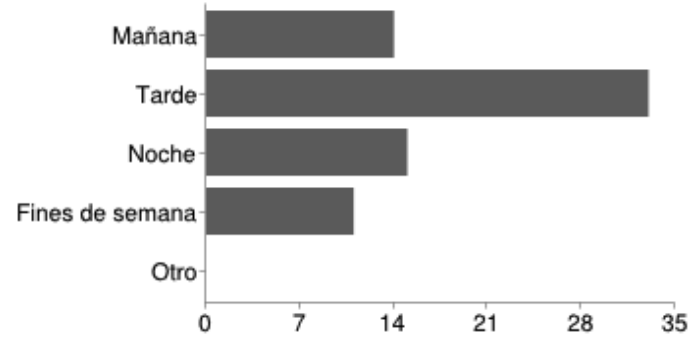

Gráfico 3: Horarios en los que se ha realizado el MOOC. Fuente: elaboración propia

En cuanto a los dispositivos de acceso, el $75 \%$ de los alumnos utilizaron el ordenador portátil para realizar el MOOC frente a otras opciones como el PC (16\%), la tableta (4\%) o el teléfono móvil (3\%).

Por último, en cuanto a la motivación y actitud demostrada por los participantes, hemos de señalar que el $100 \%$ de los encuestados recomendaría este tipo de cursos por múltiples razones. Como se muestra en el siguiente gráfico, las razones más utilizadas para justificar este interés provienen de la posibilidad de aprender a su ritmo, su gratuidad y la posibilidad de ampliar y actualizar sus conocimientos. En menor medida el prestigio del organismo que oferta el curso actúa como prescriptor.

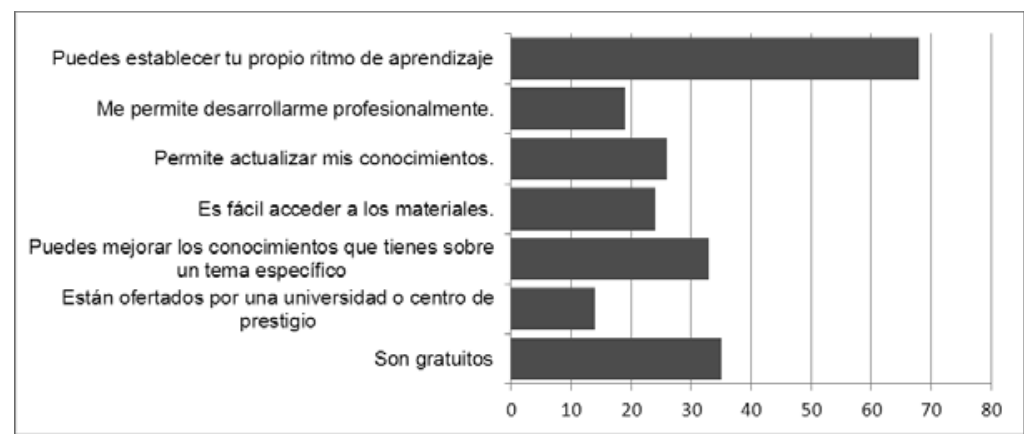

Gráfico 4: Motivos para recomendar este tipo de cursos. Fuente: elaboración propia

Este interés se constata al preguntar sobre el interés de su universidad lance una oferta de cursos de este tipo. El 100\% se muestra de acuerdo e incluso el $80 \%$ se declara dispuesto a pagar por acceder a este tipo de formación y a obtener un certificado por su participación en el mismo. No obstante, el $76 \%$ de los encuestados no pagaría más de $30 €$ por la misma, frente al $12 \%$ que no estaría dispuesto a pagar. 
No obstante, los estudiantes que previo a este experimento realizaron algún curso de estas características fueron preguntados sobre las razones que les llevaron a abandonarlo. Como podemos ver en el siguiente gráfico, la falta de conocimientos de un idioma extranjero, la falta de interés sobre el temario o el escaso valor del certificado obtenidos son los argumentos utilizados para justificar el abandono.

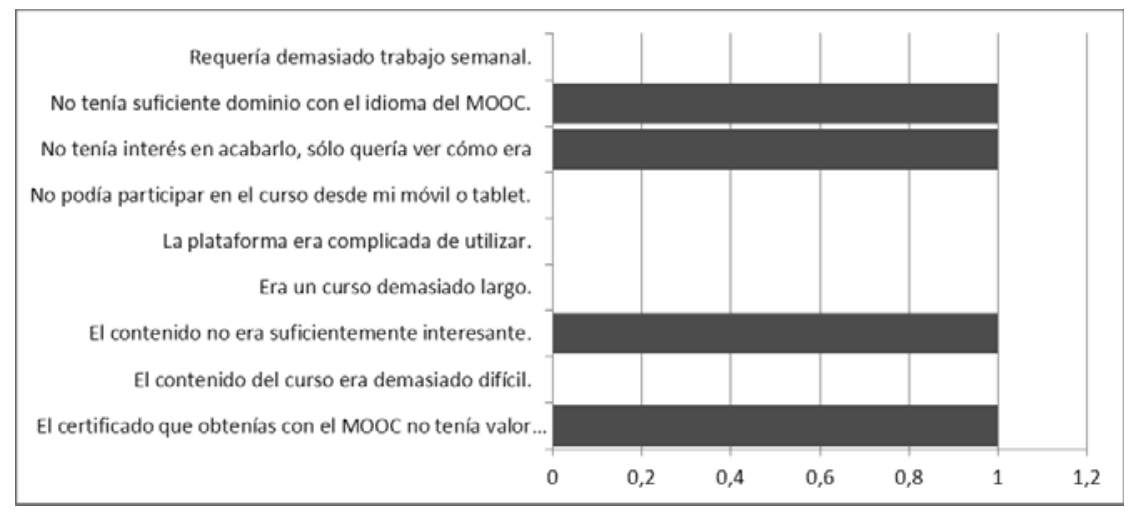

Gráfico 5: Razones por las que se ha abandonado previamente un MOOC.

Fuente: elaboración propia.

\section{Conclusiones}

Este experimento nos ha permitido obtener una información muy útil para validar a través de las opiniones de los alumnos las características de los MOOCs, anteriormente expuestas. Con este trabajo de campo hemos sido capaces de evaluar las ventajas y desventajas de este tipo de cursos. En primer lugar, hemos comprobado que se cumplen en el curso seleccionado las características propias de los MOOC: curso, accesible online, gratuito y capaz de gestionar un número amplio de estudiantes. Lamentablemente, una de las limitaciones de esta investigación nos ha hecho decantarnos por un tipo de MOOC no conectivista y, por tanto, no hemos podido evaluar la importancia y el impacto de las relaciones entre los estudiantes en la construcción del conocimiento.

Precisamente son las características de este tipo de cursos lo que los alumnos han valorado de manera más positiva. Además de ser gratuitos -algo importante dada la actual coyuntura económica-, los estudiantes han valorado muy positivamente los contenidos ofertados, muy específicos para un área de conocimiento dada, que les permite ampliar y actualizar los conocimientos adquiridos en la formación reglada tradicional. Pero quizá el aspecto mejor valorado es la posibilidad de realizar el curso a un ritmo personalizado dentro del plazo seleccionado. Esto le permite al alumnado compaginar todas sus actividades sin perder la oportunidad de seguir adquiriendo conocimientos especializados.

Sí que parece sorprendente que, al contrario de lo que sucede normalmente, los participantes no hayan considerado el prestigio de la entidad formadora como un aliciente para realizar este curso. Es precisamente el prestigio de la universidad que promueve este tipo de cursos el incentivo para conseguir un certificado que acredite el conocimiento adquirido. Este factor podría achacarse al entorno cerrado en el que se 
ha desarrollado este trabajo de campo y que no es necesariamente aplicable en todos los casos.

Aunque los resultados obtenidos nos permiten comprobar que los MOOCs son un tipo de cursos interesantes para reforzar la docencia tradicional, hay que analizar también los aspectos menos positivos. Quizá el más recurrido sea la tasa de abandono que puede verse incrementada significativamente al multiplicarse exponencialmente el número de alumnos que realizan el curso de manera simultánea. Los datos expuestos en esta investigación muestran un abandono del $20 \%$ de los estudiantes iniciales. Aunque, al no contestar el cuestionario final no tenemos la información suficiente para conocer las causas, si podemos guiarnos por la opinión de los participantes sobre cursos previos. En este caso, debemos resaltar la falta de conexión con el contenido ofertado o el idioma en el que se ofertaba como los principales obstáculos.

Otro problema importante, en este caso para las entidades promotoras de estas iniciativas, es el de la financiación. Pasada la inicial euforia que lleva a una oferta sin parangón, es necesario definir un modelo de negocio capaz de cubrir los gastos de lanzamiento y desarrollo. Hasta el momento han sido las propias instituciones las que han destinado parte de su presupuesto a fondo perdido pero, pasada la novedad el futuro de los MOOCs pasa necesariamente por la elección de un modelo de negocio que permita la captación de suficientes recursos sin ir contra su propia naturaleza, limitando el acceso a esta formación a aquellos estudiantes con mayores recursos económicos. Los datos arrojados por esta investigación permiten vislumbrar un negocio basado en el cobro de pequeñas cantidades (menos de $30 €$ ) por la certificación de los conocimientos adquiridos.

Esta investigación demuestra el interés y la potencialidad de este tipo de cursos para complementar la docencia tradicional. Las universidades tienen la oportunidad de aplicar este modelo para incrementar su productividad y posicionarse como referente de la formación continua.

\section{Referencias bibliográficas}

ATKINS, Daniel; SEELY, John; HAMMON, Allen (2007): "A Review of the Open Educational Resources (OER) Movement: Achievements, Challenges, and New Opportunities". Report to The William and Flora Hewlett Foundation. Disponible en: www.hewlett.org/uploads/files/Hewlett_OER_report.pdf [30-06-2014].

CARR, Nicholas (2012): “The crisis on Higher Education”. MIT Technology Review, vol. $115, \mathrm{n}^{\circ} 6$.

CAVANAGH, Sean (2013): “MOOCs provider targets teacher education". Education Week, Mayo 2013.

HYMAN, Paul (2012): "In the year of disruptive education". Communications of the $A C M$, vol. $55, \mathrm{n}^{\circ} 12$.

KOLOVICH, Steve (2013): “The Professors Who Make the MOOCs". Disponible en http://chronicle.com/article/The-Professors-Behind-the-MOOC/137905/\#id=overview [30-06-2014]. 
LANE, Lisa (2012): “Three kinds of MOOCs". Disponible en: http://lisahistory.net /wordpress/2012/08/three-kinds-of-moocs/

LÓPEZ, Jorge (2012). MOOC: ¿Ocaso del modelo de negocio tradicional en e-learning? Disponible en http://ojulearning.es/2012/08/mooc-ocaso-del-modelo-de-negocio-tradicional-en-e-learning/ [30-06-2014].

MOOC.ES: "cMoox vs. XMooc". Disponible en http://www.mooc.es/cmoox-vs$\mathrm{xmooc} /[30-06-2014]$.

SEELY, John (2008): "Foreword: Creating a Culture of Learning”. En: IIYOSHI, Toru and VIJAY KUMAR, M.S.: Opening Up Education: The Collective Advancement of Education Through Open Technology, Open Content, and Open Knowledge, Creative Commons edition. Princeton, New Jersey, Carnegie Foundation for the Advancement of Teaching.

ZAPATA-ROS, Miguel (2013): "MOOCs, una visión crítica. El valor no está en el ejemplar". Disponible en http://eprints.rclis.org/18452/1/MOOC_critica_Elis.pdf [11-06-2014]. 